\title{
Perda de carga contínua em tubulações conduzindo água residuária da suinocultura ${ }^{1}$
}

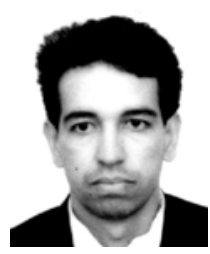

Silvio C. Sampaio², Wilson Denículi ${ }^{3}$ Rubens A. Oliveira ${ }^{3}$, Demétrius D. da Silva ${ }^{3}$, Antônio T. Matos ${ }^{3}$ \& Mauro A. Martinez ${ }^{4}$

\footnotetext{
1 Parte da Tese de Doutorado apresentada pelo primeiro autor à UFV

2 UNIOESTE/CASCAVEL. Departamento de Engenharia, Rua Universitária 2069, CEP 85814-110, Cascavel, PR. Fone: (45) 220-3000, Ramal: 3155. E-mail: ssampaio@unioeste.br (Foto)

3 DEA/UFV. CEP 36570-000, Viçosa, MG. Fone: (31) 3899-2729. E-mails: deniculi@mail.ufv.br, ralves@mail.ufv.br, david@mail.ufv.bre atmatos@mail.ufv.br

4 DEA/UFV. Fone: (31) 3899-1871. E-mail: mmauro@mail.ufv.br
}

Protocolo $092-4 / 8 / 2000$

\begin{abstract}
Resumo: O presente trabalho foi desenvolvido com o objetivo de definir e avaliar modelos matemáticos que estimem a perda de carga contínua em tubulações de aço zincado, ferro galvanizado e PVC, nos diâmetros comerciais 2 a 6 polegadas. Utilizou-se como fluido escoante a água e água residuária proveniente da suinocultura, nas concentrações de 0,98; 2,08; 4,73; 7,69 e $8,56 \%$. Os resultados mostraram que os métodos que estimam a perda de carga baseados na equação Universal e relacionam o fator de atrito às características de escoamento, não apresentaram bons ajustes, principalmente quando se utilizou o Número de Reynolds Generalizado. As equações empíricas, propostas neste trabalho, em que se utilizam as metodologias de Duffy \& Titchener e Hazen-Williams Modificada, que relacionam a perda de carga com a vazão, diâmetro, coeficiente de rugosidade e concentração de sólidos totais, apresentaram bons resultados, com coeficientes de determinação acima de 0,98. Desta maneira, recomenda-se a sua utilização para dimensionamento de projetos, por serem equações simples e confiáveis.
\end{abstract}

Palavras-chave: perda de carga, fluido não newtoniano, efluente

\section{Continuous head loss in tubes carrying swine wastewater}

\begin{abstract}
This research was performed to evaluate and introduce mathematical models to estimate head losses in zinc steel, galvanized iron and PVC tubes and diameters ranging from 2 to 6 inches. The total solid concentration of swine liquid manure was: 0.98, 2.08, 4.73, 7.69 and $8.56 \%$. The methods based on the Universal equation using the Generalized Reynolds Number showed an adjustment worse than the ones obtained through the empirical equations proposed in this work. Those equations were established from Duff \& Titchener and Modified HazenWilliams methodologies and showed a better adjustment with coefficients of determinations above 0.98 and thus may be recommended for pumping projects.
\end{abstract}

Key words: head losses, non-newtonian fluid, effluent

\section{INTRODUÇÃO}

O esterco bovino, suíno e de aves, geralmente produz grande melhoria nas propriedades físicas, biológicas e químicas do solo, devido ao seu alto teor de matéria orgânica (Hansen, 1958; Pile et al., 1975; Galbiatti et al., 1992; Edwards \& Daniel, 1993; Sekhon \& Bajwa, 1993 e Modaihsh et al., 1994); no entanto, possui alto potencial poluidor, bastante superior ao dejeto humano. Um bovino plenamente desenvolvido, por exemplo, produz a quantidade de estrume diário eqüivalente ao de 12 a 16 pessoas, levando-se em consideração a Demanda Bioquímica de Oxigênio (Chanlett, 1973). No caso de esterco suíno, esta relação é aumentada 260 vezes, segundo Oliveira (1996).

Atualmente, a utilização de águas residuárias na irrigação já representa parcela considerável. Vários trabalhos apontam que a próxima crise da humanidade estará intimamente relacionada com a disponibilidade e conservação dos recursos hídricos. Sabe-se que a água, apesar de ser um recurso renovável, é limitada devido à sua má distribuição espacial e temporal.

A utilização de águas residuárias na irrigação deverá exercer fator econômico fundamental para o irrigante, já que a irrigação representa cerca de $80 \%$ da água consumida, como demonstram os trabalhos realizados pela Centrais Elétricas Minas Gerais (CEMIG) e nos Estados Unidos e Rússia (Lanna, 1993). É 
importante salientar que, no futuro, a água captada nos lagos e rios passará a ser taxada devido à implantação da nova legislação sobre o uso da água em todos os estados brasileiros.

Sabe-se que a irrigação, quando realizada com águas residuárias, opera com características hidráulicas do escoamento nas tubulações praticamente desconhecidas, podendo estar levando técnicos a cometerem erros consideráveis no seu dimensionamento. Existem poucos trabalhos que abordam o assunto com detalhe, devido talvez ao grau de complexidade envolvido, por se tratar de um fluido com comportamento diferente da água.

Deste modo e visando obter informações específicas sobre tal comportamento, este trabalho teve por objetivo definir e avaliar equações matemáticas que estimem a perda de carga distribuída em tubulações comerciais, operando com água residuária da suinocultura (ARS).

\section{MATERIAL E MÉTODOS}

A partir dos dados obtidos por Sampaio (1999) realizaram-se análises estatísticas de regressões lineares e não lineares, tendo em vista um modelo matemático de melhor ajuste. $\mathrm{O}$ trabalho do autor, conduzido na Universidade Federal de Viçosa, MG, determinou as perdas de carga ocorridas nas tubulações de aço zincado, com diâmetros de 73,54; 99,83; 125,24 e 150,43 mm, ferro galvanizado, com diâmetros de 53,75; 84,01; 105,90; 130,30 e 155,58 mm, e PVC, com diâmetros de 52,61; 79,90; 103,22; 127,31 e 153,43 mm. Os fluidos escoantes nessas tubulações foram a água e a ARS, nas concentrações de 0,$98 ; 2,08 ; 4,73$; 7,69 e $8,56 \%$ de sólidos totais.

Deste modo, a partir dos dados obtidos para as variáveis: perda de carga, concentração de sólidos totais, vazão, material de fabricação da tubulação (coeficiente C de Hazen-Williams) e diâmetros, avaliou-se os modelos de equações de perda de carga, descritos a seguir.

\section{Modelo de Chen \& Hashimoto (1976b)}

Os autores ajustaram o modelo de Blasius (Eq. 1) utilizando, como fluido, as águas residuárias da bovinocultura, suinocultura e avicultura, nas concentrações de 3 a $5 \%$, e o Número de Reynolds Generalizado, na faixa de 4300 a 100000.

Foi ajustado um único modelo para todas as concentrações de sólidos totais e tubulações estudados, em que se utilizou o trabalho de Hashimoto \& Chen (1976) para determinar as propriedades reológicas do esterco.

$$
f=\operatorname{aReg}^{b}
$$

em que:

a, b - constantes características do fluido, adimensionais

Reg - Número de Reynolds Generalizado, adimensional

O número de Reynolds Generalizado foi determinado pela equação:

$$
\operatorname{Reg}=\frac{D^{n}\left(V^{2-n}\right) \rho}{8^{n-1} K}\left(\frac{4 n}{1+3 n}\right)^{n}
$$

em que:

D - diâmetro interno da tubulação, $m$

$\mathrm{V}$ - Velocidade de escoamento, $\mathrm{m} \mathrm{s}^{-1}$

$\rho \quad$ - massa específica do fluido, $\mathrm{kg} \mathrm{m}^{-3}$

$\mathrm{K}, \mathrm{n}$ - propriedades reológicas do fluidos, adimensionais

As propriedades reológicas de um fluido são representadas pelas constantes K e n da Equação Geral da Viscosidade, segundo Hughes \& Brigthon (1974) e Bourne (1982). Buscou-se determinar essas propriedades em laboratório para a água residuária da suinocultura, porém o reômetro utilizado apresentou dados inconsistentes, provavelmente em função de entupimentos e travamento ocorridos durante os testes. Assim, do trabalho de Chen \& Hashimoto (1976a) retirou-se os valores dessas constantes.

\section{Modelo de Dodge \& Metzner}

Este modelo, que teve como base a fórmula de von Kármán, representado pela Eq. 3, relaciona o fator $\mathrm{f}$ de atrito com o Número de Reynolds Generalizado, no regime de escoamento turbulento liso (Hughes \& Brighton, 1974).

Seguindo-se a recomendação dos autores, determinou-se uma equação para cada tipo e concentração de sólidos totais de ambos os estercos líquidos.

$$
\frac{1}{\mathrm{f}}=\frac{4}{\mathrm{n}^{0,75}} \log \left[\operatorname{Reg}(\mathrm{f})^{1-\frac{\mathrm{n}}{2}}\right]-\frac{0,4}{\mathrm{n}^{1,2}}
$$

em que:

f - fator de atrito da Equação Universal, adimensional

\section{Modelo de Lee \& Duffy (1976)}

Também com base na equação de von Kármán, trata o fluido não newtoniano como newtoniano, relacionando o número de Reynolds com o fator $\mathrm{f}$ de atrito. Este modelo é representado pela Eq. 4.

Foram ajustados, para as tubulações de aço zincado, ferro galvanizado e PVC e as respectivas concentrações de ARS, resultando um número de 15 equações.

$$
\frac{1}{\sqrt{\mathrm{f}}}=\frac{1}{\beta} \operatorname{Ln}(\operatorname{Rey} \sqrt{\mathrm{f}})+\left(14-\frac{5,6}{\beta}\right)
$$

em que:

$\beta \quad$ - constante característica do fluido, adimensional

Rey - Número de Reynolds, adimensional

Ressalta-se que o número de Reynolds (Rey) corresponde ao número de Reynolds Generalizado (Reg) quando se considera o fluido newtoniano, isto é, $\mathrm{n}=1 \mathrm{e} \mathrm{K}=\mu$ (em que: $\mu$ - viscosidade dinâmica do fluido).

\section{Modelo de Lee \& Duffy (1976) Modificado}

Praticamente, consiste no próprio modelo anterior, proposto aqui com pequenas alterações na sua forma, como demonstra a Eq. 5. Procurou-se, com essas modificações, um ajuste melhor com os dados levantados. 


$$
\frac{1}{\sqrt{f}}=a(\ln \operatorname{Re} y)(\sqrt{f})+b
$$

\section{em que:}

a e b - constantes características do fluido, adimensionais

\section{Modelo de Duffy \& Titchener (1974)}

É um modelo empírico que estima a perda de carga em função da velocidade, do diâmetro e da concentração de sólidos totais para cada tipo de material de tubulação (Eq. 6). Deste modo, foi ajustada uma equação ao aço zincado, ferro galvanizado e PVC.

$$
\mathrm{J}=\beta_{1} \mathrm{~V}^{\beta_{2}} \mathrm{ST}^{\beta_{3}} \mathrm{D}^{\beta_{4}}
$$

em que:

$\mathrm{J}$ - perda de carga unitária, $\mathrm{mm}^{-1}$

ST - concentração de sólidos totais, \%

D - diâmetro interno da tubulação, $m$

$\beta_{1}, \beta_{2}, \beta_{3}$ e $\beta_{4}$ - constantes características do fluido, adimensionais

\section{Modelo de Hazen-Williams Modificado}

É uma nova proposta do modelo de Hazen-Williams, adaptado para ARS (Eq. 7). Verifica-se que este modelo, semelhante à equação de Hazen-Williams, possui grande potencial de uso devido a sua simplicidade e capacidade de síntese.

$$
J=\frac{k 1(S T)^{k_{2}} Q^{k_{3}}}{C^{k_{4}} D^{k_{5}}}
$$

em que:

C - coeficiente que depende da natureza das paredes do tubo e do seu estado de conservação, admensional

Q - vazão do fluido, $\mathrm{m}^{3} \mathrm{~s}^{-1}$

D - diâmetro interno da tubulação, $m$

$\mathrm{k}_{1}, \mathrm{k}_{2}, \mathrm{k}_{3}, \mathrm{k}_{4}$ e $\mathrm{k}_{5}$ - parâmetros de ajuste da equação, adimensionais

Ressalta-se que os valores de $\mathrm{C}$ das tubulações utilizadas na modelagem desta equação, são médias aritméticas de 10 observações, determinadas a partir dos dados levantados em campo e estimados por meio da equação de Hazen-Williams.

\section{RESULTADOS E DISCUSSÃO}

O modelo em que se utilizou a metodologia proposta por Chen \& Hashimoto (1976b) e as constantes da equação de Chen \& Hashimoto (1976a) encontra-se na Tabela 1.

$\mathrm{Na}$ determinação da equação apresentada por Chen \& Hashimoto (1976a) para águas residuárias da bovinocultura e suinocultura, os autores usaram apenas dois tubos de alumínio de 48 e 74,2 mm de diâmetro encontrando, assim, não mais que 50 observações. No presente trabalho, o número de observações foi de 331 para a ARS, englobando dados obtidos para tubulações de aço zincado, ferro galvanizado e PVC, com
Tabela 1. Equação de estimativa do fator $\mathrm{f}$ de atrito em função do Número de Reynolds Generalizado (Reg) para o ARS*, seguindo-se a metodologia de Chen \& Hashimoto (1976b)

\begin{tabular}{ccc}
\hline $\begin{array}{c}\text { Faixa de Concentração de } \\
\text { Sólidos Totais para ARS }\end{array}$ & Equação & $\mathrm{R}^{2}$ \\
\hline$(2,08$ a $7,69 \%)$ & $\mathrm{f}=0,1169 \mathrm{Reg}^{-0,1687}$ & 0,5108 \\
\hline * Águas residuárias de suinocultura & &
\end{tabular}

diâmetros na faixa de 50 a $150 \mathrm{~mm}$ e três concentrações de sólidos totais.

A equação da Tabela 1, foi encontrada utilizando-se apenas os dados que se encontravam dentro dos limites do Número de Reynolds Generalizado (4300 a 100000) sugeridos por Chen \& Hashimoto (1976b). Deste modo, como várias observações foram descartadas, a faixa de concentração de sólidos totais usada na determinação dessas equações, citada nesta Tabela, não englobou toda aquela faixa levantada em campo $(0,98$ a $8,56 \%)$. Portanto, a equação apresentada nesta tabela é válida para faixa de concentração de sólidos totais apresentada e para qualquer um dos três materiais de fabricação das tubulações avaliadas.

Observou-se que dos parâmetros de ajuste encontrados nesta equação apenas o expoente aproximou-se bastante daquele apresentado por Chen \& Hashimoto (1976b), que foi de $-0,18$. Enquanto que a constante linear da equação encontrada por esses autores foi 0,030 .

O baixo coeficiente de determinação encontrado, apesar da equação ser estatisticamente significativa ao nível de $1 \%$, como demonstrou a análise de regressão (teste F), ocorreu provavelmente, devido aos dados utilizados de $\mathrm{K}$ e $\mathrm{n}$ (constantes reológicas) obtidos a partir do trabalho de Chen \& Hashimoto (1976a), que não caracterizaram satisfatoriamente a água residuária utilizada neste trabalho. Fato este evidenciado quando se observa o diferente valor encontrado das constantes reológicas para água residuária da bovinocultura, por Kumar et al. (1972), Chen \& Hashimoto (1976a) e Bashford et al. (1977), pois demonstra como essas constantes podem sofrer variações em função dos mais diversos fatores.

Resultados encontrados quando se utilizou o método de Dodge \& Metzner, citados por Hughes \& Brighton (1974) foram descartados quando se avaliaram os seus ajustes com os dados observados confirmando, assim, a citação de Chen \& Hashimoto (1976b) de que os estercos líquidos não são, em geral, pseudoplásticos típicos.

O método de Lee \& Duffy (1976) tem, como característica, ajustar o fator $\mathrm{f}$ de atrito ao número de Reynolds, tratando o fluido como se fosse a água. Deste modo, esperava-se melhor ajuste que os métodos propostos citados anteriormente; como isto não ocorreu, estudou-se um outro modelo matemático, com base nas afirmações dos autores, denominado Lee \& Duffy (1976) Modificado.

Os resultados desta metodologia, apresentados na Tabela 2 , mostraram que o ajuste encontrado pode ser considerado satisfatório estatisticamente, como mostrou a análise de regressão, na qual os testes indicaram que os coeficientes de ajuste e de determinação de todas as equações foram significativos ao nível de $1 \%$, apesar de alguns valores dos coeficientes angulares serem relativamente baixos. 
Tabela 2. Equações de estimativas do fator f de atrito em função do número de Reynolds (Rey) para as respectivas concentrações da água residuária da suinocultura (ARS), seguindo-se a metodologia Lee \& Duffy (1976) Modificado

\begin{tabular}{|c|c|c|c|}
\hline Material & $\begin{array}{c}\text { Concentração de } \\
\text { Sólidos Totais (\%) }\end{array}$ & Equação & $\mathrm{R}^{2}$ \\
\hline \multirow{5}{*}{$\begin{array}{c}\text { Aço } \\
\text { Zincado }\end{array}$} & 0,98 & $1 / \sqrt{f}=-4,095 \operatorname{Ln}($ Rey $) \sqrt{f}+15,776$ & 0,6497 \\
\hline & 2,08 & $1 / \sqrt{\mathrm{f}}=-3,8468 \operatorname{Ln}($ Rey $) \sqrt{\mathrm{f}}+14,825$ & 0,8777 \\
\hline & 4,73 & $1 / \sqrt{f}=-3,0167 \operatorname{Ln}(\operatorname{Rey}) \sqrt{f}+13,121$ & 0,8960 \\
\hline & 7,69 & $1 / \sqrt{\mathrm{f}}=-2,5923 \operatorname{Ln}($ Rey $) \sqrt{\mathrm{f}}+12,144$ & 0,8927 \\
\hline & 8,56 & $1 / \sqrt{\mathrm{f}}=-2,2467 \operatorname{Ln}($ Rey $) \sqrt{\mathrm{f}}+11,270$ & 0,8854 \\
\hline \multirow{5}{*}{$\begin{array}{c}\text { Ferro } \\
\text { Galvanizado }\end{array}$} & 0,98 & $1 / \sqrt{\mathrm{f}}=-3,934 \operatorname{Ln}($ Rey $) \sqrt{\mathrm{f}}+14,873$ & 0,5399 \\
\hline & 2,08 & $1 / \sqrt{\mathrm{f}}=-3,5407 \operatorname{Ln}($ Rey $) \sqrt{\mathrm{f}}+14,181$ & 0,8610 \\
\hline & 4,73 & $1 / \sqrt{\mathrm{f}}=-2,9756 \operatorname{Ln}($ Rey $) \sqrt{\mathrm{f}}+13,016$ & 0,8707 \\
\hline & 7,69 & $1 / \sqrt{\mathrm{f}}=-2,8386 \operatorname{Ln}(\operatorname{Rey}) \sqrt{\mathrm{f}}+12,711$ & 0,8193 \\
\hline & 8,56 & $1 / \sqrt{\mathrm{f}}=-2,3322 \operatorname{Ln}($ Rey $) \sqrt{\mathrm{f}}+11,503$ & 0,8521 \\
\hline \multirow{5}{*}{ PVC } & 0,98 & $1 / \sqrt{\mathrm{f}}=-4,6067 \operatorname{Ln}($ Rey $) \sqrt{\mathrm{f}}+16,178$ & 0,7417 \\
\hline & 2,08 & $1 / \sqrt{\mathrm{f}}=-4,0639 \operatorname{Ln}($ Rey $) \sqrt{\mathrm{f}}+15,223$ & 0,8360 \\
\hline & 4,73 & $1 / \sqrt{\mathrm{f}}=-3,6903 \operatorname{Ln}($ Rey $) \sqrt{\mathrm{f}}+14,496$ & 0,8146 \\
\hline & 7,69 & $1 / \sqrt{\mathrm{f}}=-3,2002 \operatorname{Ln}($ Rey $) \sqrt{\mathrm{f}}+13,531$ & 0,8177 \\
\hline & 8,56 & $1 / \sqrt{\mathrm{f}}=-2,9517 \operatorname{Ln}($ Rey $) \sqrt{\mathrm{f}}+12,917$ & 0,6859 \\
\hline
\end{tabular}

Este método é justificado pelas citações de Arney et al. (1993), Huang et al. (1993) e Joseph et al. (1997) em que o modelo de Blasius pode ser muito simples para caracterizar todo o comportamento do líquido não newtoniano. Hemström et al. (1976) citam ainda que, quando são desconhecidas as propriedades reológicas e/ou o modelo matemático adotado não apresenta bons resultados, é necessário encontrar-se uma equação que caracterize bem o escoamento do fluido.

Verifica-se que todos os módulos dos coeficientes angulares e lineares das equações encontradas nesta metodologia diminuem com o aumento da concentração de sólidos totais; fica evidente, portanto, que esses coeficientes são característicos de cada concentração de sólidos totais e materiais de fabricação da tubulação.

Pode-se observar que os modelos obtidos a partir da metodologia apresentada por Duffy \& Titchener (1974) apresentaram melhores ajustes que os anteriores (Tabela 3).

Os expoentes da velocidade e diâmetro estão dentro da faixa apresentada por Duffy (1976) para diferentes fluidos escoantes não newtonianos e se aproximam daqueles apresentados por Hazen \& Williams (1963) que são 1,85 e 1,17, respectivamente.
Obteve-se a Eq. $8\left(\mathrm{R}^{2}=0,9860\right)$, quando se fez uso do modelo de Hazen-Williams Modificado. Nota-se que, neste caso, apenas o expoente do coeficiente de rugosidade (C) não se aproxima daqueles apresentados por Hazen \& Williams (1963) como acontece com os expoentes da vazão $(\mathrm{Q})$ e do diâmetro (D). Isto demonstra que, provavelmente, exista uma interação entre o tipo de água residuária avaliada e suas concentrações de sólidos totais com a rugosidade da tubulação.

$$
J=\frac{0,540257 \mathrm{ST}^{0,173681} \mathrm{Q}^{1,789577}}{\mathrm{C}^{1,172486} \mathrm{D}^{4,58967}}
$$

Este fato não se torna, porém, uma regra geral para fluidos não newtonianos, como demonstram os resultados de Duffy (1976) e Duffy \& Titchener (1974), em que cada fluido não newtoniano apresentou valores diferentes dos expoentes de vazão e diâmetro. Provavelmente, quanto maior a diferença desses expoentes com aqueles apresentados por Hazen \& Williams (1963) mais o fluido apresenta características de não newtoniano.

Tabela 3. Equações que estimam a perda de carga - J $\left(\mathrm{m} \mathrm{m}^{-1}\right)$ em função da velocidade de escoamento - V ( $\left.\mathrm{m} \mathrm{s}^{-1}\right)$, concentração de sólidos totais - ST (\%) e diâmetro - D (m) para todos os materiais avaliados e águas residuárias da suinocultura (ARS), seguindo-se a metodologia de Duffy \& Titchener (1974)

\begin{tabular}{ccc}
\hline Material & Equação & $\mathrm{R}^{2}$ \\
\hline Aço Zincado & $\mathrm{J}=0,000680 \mathrm{~V}^{1,804093} \mathrm{ST}^{0,240494} \mathrm{D}^{-1,10413}$ & 0,9937 \\
Ferro Galvanizado & $\mathrm{J}=0,0009651 \mathrm{~V}^{1,841604} \mathrm{ST}^{0,181387} \mathrm{D}^{-0,996181}$ & 0,9909 \\
PVC & $\mathrm{J}=0,000773 \mathrm{~V}^{1,781414} \mathrm{ST}^{0,116384} \mathrm{D}^{-1,08216}$ & 0,9948 \\
\hline
\end{tabular}


Tabela 4. Comparação entre as metodologias desenvolvidas e os dados observados para águas residuárias da suinocultura (ARS)

\begin{tabular}{|c|c|c|c|c|c|c|c|c|c|c|}
\hline \multirow{2}{*}{$\begin{array}{l}\text { Material da } \\
\text { Tubulação }\end{array}$} & \multirow{2}{*}{$\begin{array}{c}\mathrm{C} \\
\text { Médio }\end{array}$} & \multirow{2}{*}{$\begin{array}{l}\text { Diâmetro } \\
\text { (m) }\end{array}$} & \multirow{2}{*}{$\begin{array}{l}\text { Velocidade } \\
\left(\mathrm{m} \mathrm{s}^{-1}\right)\end{array}$} & \multirow{2}{*}{$\begin{array}{l}\text { ST } \\
(\%)\end{array}$} & \multicolumn{6}{|c|}{ Perda de Carga* m $(1000 \mathrm{~m})^{-1}$} \\
\hline & & & & & 1 & 2 & 3 & 4 & 5 & 6 \\
\hline \multirow{3}{*}{$\begin{array}{c}\text { Aço } \\
\text { Zincado }\end{array}$} & 164 & 0,073540 & 1,79028 & 0,98 & 35,61 & -- & 64,56 & 37,12 & 35,03 & 34,01 \\
\hline & 170 & 0,099825 & 1,09093 & 4,73 & 12,67 & 14,42 & 18,96 & 14,10 & 13,36 & 11,94 \\
\hline & 160 & 0,150430 & 0,97706 & 7,69 & 10,56 & 8,82 & 8,72 & 8,62 & 8,47 & 4,72 \\
\hline \multirow{3}{*}{$\begin{array}{c}\text { Ferro } \\
\text { Galvanizado }\end{array}$} & 155 & 0,084000 & 2,02629 & 8,56 & 67,61 & 59,54 & 58,63 & 64,89 & 59,51 & 40,67 \\
\hline & 158 & 0,105900 & 1,50735 & 2,08 & 21,33 & 18,78 & 73,91 & 20,85 & 21,21 & 17,28 \\
\hline & 147 & 0,155750 & 0,78937 & 4,73 & 5,56 & 3,56 & 5,77 & 4,95 & 5,66 & 3,33 \\
\hline \multirow{3}{*}{ PVC } & 164 & 0,052600 & 2,74326 & 2,08 & 124,78 & -- & 125,59 & 120,73 & 120,23 & 81,17 \\
\hline & 164 & 0,103200 & 1,61751 & 0,98 & 19,33 & -- & 19,43 & 21,13 & 20,74 & 20,00 \\
\hline & 146 & 0,153400 & 0,28353 & 7,69 & 0,78 & -- & 1,16 & 0,80 & 1,01 & 0,61 \\
\hline
\end{tabular}

*1 - Dados de campo; 2 - Chen \& Hashimoto (1976b); 3 - Lee \& Duffy Modificada; 4 - Duffy \& Titchener (1974); 5 - Hazen-Williams Modificada; 6 - Dados de campo para Água

Para os dois modelos avaliados o número de observações considerado foi de 200 para as tubulações de aço zincado e de 230 para as tubulações de ferro galvanizado e PVC, pois o número de diâmetros estudados foi diferente, além de não ter sido possível a avaliação dos menores diâmetros das tubulações de ferro galvanizado e PVC, devido ao entupimento ocorrido quando se trabalhou com as duas maiores concentrações de sólidos totais, segundo Sampaio (1999). Logo, não se recomenda o uso desses modelos para tubulações com diâmetros de $50 \mathrm{~mm}$, aproximadamente, quando o fluido escoante for ARS com concentração de sólidos totais acima de $7,69 \%$.

Os expoentes dos diâmetros e velocidades das equações encontradas, estão próximos das faixas citadas por Azevedo Neto (1982) de 1,3 a 1,0 e 1,7 a 2,0, respectivamente, determinadas a partir das experimentações realizadas por diversos pesquisadores, utilizando a água como fluido escoante. Segundo o autor, uma fórmula considerada racional e com base nessas considerações e na análise dimensional, deve possuir expoentes situados próximos às faixas citadas.

De modo a comprovar a precisão das metodologias que apresentaram os melhores ajustes, são apresentados, na Tabela 4 , os resultados estimados e observados, escolhidos ao acaso.

As estimativas que mais se aproximaram dos dados observados correspondem às equações de melhor ajuste, destacando-se as metodologias de Duffy \& Titchener (1974) e Hazen-Williams Modificada, enquanto a metodologia de Chen \& Hashimoto (1796b) possui grande limitação, devido à faixa recomendada para sua aplicação $(4300 \leq \operatorname{Reg} \leq 100000)$.

Nota-se, também que a recomendação de Estep, citado por Kumar et al. (1972) para se adicionar 10\% na perda de carga, visando ao bombeamento com estercos líquidos, é válida apenas para as concentrações inferiores. Esta diferença na perda de carga, entre água e ARS, pode atingir valores próximos a $100 \%$, para as maiores concentrações avaliadas.

Comprova-se facilmente que os resultados apresentados neste trabalho corroboram com a citação de Duffy (1976) de que os modelos empíricos em geral apresentam melhores resultados que os modelos que estimam o fator $\mathrm{f}$ de atrito.

Segundo Azevedo Neto (1982) os resultados obtidos utilizando-se as 110 fórmulas práticas encontradas na literatura para determinação da perda de carga para água, chegam a ter uma variação superior a $100 \%$. Deste modo, a ponderação realizada por Fanning, citado por Azevedo Neto (1982) é bastante relevante: "Graves erros podem provir do uso pouco racional e inconveniente das fórmulas. $\mathrm{O}$ conhecimento completo da origem de uma fórmula é essencial para a segura aplicação prática".

\section{CONCLUSÕES}

De acordo com os resultados encontrados, conclui-se que:

1. Os métodos que estimam a perda de carga, com base na Equação Universal e relacionam o fator de atrito às características de escoamento, não apresentaram bons ajustes, principalmente quando se utilizou o Número de Reynolds Generalizado.

2. As equações empíricas propostas neste trabalho, utilizando-se as metodologias de Duffy \& Titchener (1974) e Hazen-Williams Modificada e que relacionam a perda de carga com a vazão, diâmetro, coeficiente de rugosidade e concentração de sólidos totais, apresentaram bons resultados, com coeficientes de determinação acima de $98 \%$. Desta maneira, recomenda-se a sua utilização para dimensionamento de projetos, por serem equações simples e confiáveis.

\section{AGRADECIMENTOS}

O primeiro autor agradece ao $\mathrm{CNPq}$ que financiou a pesquisa por meio da bolsa de Doutorado, e ao Laboratório de Hidráulica da UFV, que cedeu todas as tubulações e reservatório utilizados na condução deste trabalho.

\section{LITERATURA CITADA}

Arney, M.S; Bai, R; Guevara E; Joseph, D.D; Liu, K. Friction factor and holdup studies for lubricated pipelining experiments and correlations. Journal Multiphase Flow, London, v.19, n.6, p.1061-1076, 1993.

Azevedo Neto, J.M. Manual de hidráulica. $7^{\mathrm{a}}$ ed., São Paulo: Edgard Blücher Ltda, 1982. 336p.

Bashford, L.L; Gilbertson, C.B; Nienaber, J.A; Tietz, D. Effects of ration roughage content on viscosity and theoretical head losses in pipe flow for beef cattle slurry. Transactions of the ASAE, St. Joseph, v. 20, n. 6, p.1106-1109, 1977.

Bourne, M.C. Food texture and viscosity (concept and management). New York:Academic Press, 1982.325p.

Chanlett, E.T. Environmental protection. New York: McGraw Hill, 1973. 569p. 
Chen, Y.R; Hashimoto, A.G. Rheological properties of aerated poultry waste slurries. Transactions of the ASAE, St. Joseph, v.19, n.5, p.128-133, 1976a.

Chen, Y.R; Hashimoto, A.G. Pipeline transport of livestock waste slurries. Transactions of the ASAE, St. Joseph, v.19, n.5, p.898-906, 1976b.

Duffy, G.G. A review and evaluation of design methods for calculating friction loss in stock piping systems. Tappi, Atlanta, v.59, n.8, p.124-127, 1976.

Duffy, G.G; Titchener A.L. Design procedures for obtaining pipe friction loss for chemical pulps. Tappi, Atlanta, v.57, n.5, p.162-166, 1974.

Edwards, D.R; Daniel, T.C. Abstractions and runoff from fescue plots receiving poultry litter and swine manure. Transactions of the ASAE, St. Joseph, v.36, n.2, p.405-411, 1993.

Galbiatti, J.A; Castellane, P.D; Garcia A. Efeito da irrigação e de adubação mineral e orgânica no desenvolvimento e produtividade da cebola, cultivar piralopes. Revista Cientifica-Jaboticabal, Jaboticabal, v. 20, n.2, p.371-378, 1992

Hansen, C.M. Engineering principles in handling liquid materials. Agricultural Engineering, St. Joseph, v.19, n.5, p.546-551, 1958.

Hashimoto, A.G; Chen, Y.R. Rheology of livestock waste slurries. Transactions of the ASAE, St. Joseph, v.19, n.5, p.930-934, 1976.

Hazen, A; Williams, G.S. Hydraulic tables. New York: John Wiley \& Sons, 1963. 120p.

Hemström, G; Moller, K; Norman, B. Boundary layer studies in pulp suspension flow. Tappi, Atlanta, v.59, n.8, p.115-118, 1976.

Huang, A; Christodoulou, C; Joseph, D.D. Friction factor and holdup studies for lubricated pipelining: Laminar and models of eccentric core flow. Journal Multiphase Flow, London, v.19, n.6, p.481-497, 1993.
Hughes, W.F; Brighton, J.A. Dinâmica dos fluidos. São Paulo: McGraw-Hill, 1974. 358p.

Joseph, D.D; Bai, R; Chen K.P; Renardy Y.Y. Core-annular flows. Annual Review of Fluid Mechanics, New York, v.29, p.401-408, 1997.

Kumar, M; Bartelett, H.D; Mohsenin, N.N. Flow properties of animal waste slurries. Transactions of the ASAE, St. Joseph, v.30, n.2, p.718-722, 1972.

Lanna, A.E. Gestão dos recursos hídricos. In: Tucci, E. Hidrologia - ciência e aplicação. Porto Alegre: ABRH: EDUSP, 1993. p.727-804.

Lee, P.F.W; Duffy, G.G. An analysis of the drag reducing regime of pulp suspension flow. Tappi, Atlanta, v.59, n.8, p.119-123, 1976.

Modaihsh, A.S; Al Mustafa, W.A; Abdallah, A.E; El Shall, A.A. Impact of sulfate-rich water and organic manures on wheat grown in arid soils. Arid Soil Research and Rehabilitation, Logan, v.8, n.3, p.247-259, 1994.

Oliveira, R.A. Desempenho de bomba centrífuga operando com esterco bovino líquido. Viçosa: UFV, 1996. 73p. Tese Doutorado

Pile, R.S; Wills, J.B; Sewell, J.I. Effects of furrow irrigation with water and dairy manure slurry on corn yield. Tennessee Farm and Home Science, Tennessee, n.548, p.24-26. 1975.

Rouse, H. Modern conceptions of the mechanics of fluid turbulence. Classic Paper in Hydraulics. ASAE, New York, p.52-94. 1982.

Sampaio, S.C. Perda de carga em tubulações comerciais utilizando como fluidos circulantes águas residuárias da bovinocultura e suinocultura. Viçosa: UFV, 1999. 168p. Tese Doutorado

Sekhon, B.S; Bajwa, M.S. Effect of organic matter and gypsum in controlling soil sodicity in a rice-wheat-maize system irrigated with sodic waters. Agricultural-Water-Management, Amsterdam v. 24, n.1, p.15-25, 1993. 